Nisho-02/1

\title{
PseudoSkyrmion Effects on Tunneling Conductivity in Coherent Bilayer Quantum Hall States at $\nu=1$
}

\author{
Aiichi Iwazaki \\ Department of Physics, Nishogakusha University, Shonan Ohi Chiba 277, Japan.
}

(March 1, 2002)

\begin{abstract}
We present a mechamism why interlayer tunneling conductivity in coherent bilayer quantum Hall states at $\nu=1$ is anomalously large, but finite in the recent experiment. According to the mechanism, pseudoSkyrmions causes the finite conductivity, although there exists an expectation that dissipationless tunneling current arises in the state. PseudoSkyrmions have an intrinsic polarization field perpendicular to the layers, which causes the dissipation. Using the mechanism we show that the large peak in the conductivity remains for weak parallel magnetic field, but decay rapidly after its strength is beyond a critical one, $\sim 0.1$ Tesla.
\end{abstract}

PACS 73.43.-f,73.21.-b,71.10.Pm,12.39.Dc

Quantum Hall Effects, Skyrmion, Interlayer Phase Coherence

Typeset using REVTEX 
It has recently been observed [1] that interlayer tunneling conductivity in bilayer quantum Hall states at $\nu=1$ shows anomalous zero bias peak when layer distance $d$ is comparable with magnetic length $l=\sqrt{1 / e B}$. This suggests [2] that the condensation of exitonic excitation ( a pair of an electron in a layer and a hole in the other layer ) is realized and the interlayer phase coherence between the two layers comes out. Subsequent observations [3] of NambuGoldstone mode and quantized Hall drag have strongly supported this intriguing feature «4 in the bilayer quantum Hall states.

It is well known that quantum Hall states in a single layer can be understood as a condensed state [5] of bosonized electrons [6] with a single spin degree of freedom. In the state, however, there is no physically relevant phase associated with the bosonized electrons because there exists a Chern-Simons gauge symmetry which can make the phase vanish. On the other hand, in the bilayer quantum Hall states there are electrons with two degree of freedom, i.e. pseudo-Spin. We have, in general, two Chern-Simons gauge symmetries [7] corresponding to bosonization of these two types of electrons. They can also make the phases of the electrons vanish. Hence, there are no physically relevant phases. But some of the bilayer quantum Hall states [4] are described only by using a single Chern-Simons gauge symmetry. The gauge symmetry rotates the phase of each type of bosonised electrons identically. Thus, the difference of the two phases can not be made to vanish so that it is a physically relevant variable. This physically relevant phase difference comes out as a result of the condensation of the exitons. In this way the bilayer quantum Hall states with the interlayer phase coherence are realized [4]. Especially, the states at total filling factor $\nu_{1}+\nu_{2}=1$ are under theoretical and experimental investigation at present.

It has been recognized [8,9] that these bilayer quantum Hall states possesses various interesting features corresponding to parameters present in this system, parallel magnetic field $B_{\|}$, layer distance $d$, imbalance $\sigma=\left(\rho_{1}-\rho_{2}\right) /\left(\rho_{1}+\rho_{2}\right)$ of electron densities $\rho_{i}$ between the layers, etc; the index $i$ denotes $i$-th layer. Among them, the interlayer phase coherence associated with the condensation of electron-hole pairs is the most intriguing feature of the 
states realized by choosing parameters $d \simeq l(=\sqrt{1 / e B})$ at filling factor $2 \pi \rho / e B=1($ $\left.\rho=\rho_{1}+\rho_{2}\right)$. The state is stable against with changing charge imbalance. This feature is associated with the indefiniteness of the electron number difference in the state, which is a result of the exiton condensation. Furthermore, the energies of excitations in the state with $\sigma \simeq 0$ decrease rapidly with the parallel magnetic field, while those of excitations in the state with $\sigma \simeq 1$ i.e. in a single layer, increase with $B_{\|}$10,11. The decrease of the excitation energy is a property of pseudoSkyrmion [10], which is topological excitation with pseudo-spin. On the other hand, the increase of the excitation energy is that of Skyrmion [11] with real spin.

In the observation of the interlayer tunneling conductance around $\sigma \simeq 0$, the pseudoSkyrmions are relevant excitations and may affect seriously on the tunneling current. Although we expect the presence of dissipationless tunneling current [4] in the state with the phase coherence, any small disturbance of the phase coherence caused by such excitations may give rise dissipation in the tunneling current.

In this letter we point out that pseudoSkyrmions induce the dissipation of the energy of the tunneling current $\vec{J}$. The point is that the pseudoSkyrmion has a polarization field $\vec{E}_{p}$ associated with it's polarized electric charge distribution so that the dissipation $\int \vec{E}_{p} \vec{J} \neq 0$ occurs. The dissipation by the pseudoSkyrmion only arises in the states with the imbalance of the charge densities, $\sigma \neq 0$. In other words, the dissipation by the pseudoSkyrmions does not arise by adjusting exactly the parameter such as $\sigma=0$.

Let us first derive briefly the Josephson equation in the presence of the condensation [4,9], $<\Psi_{1}^{\dagger} \Psi_{2}>\propto e^{-i \theta} \neq 0$, of the exitons. Hereafter, we consider only the quantum Hall states with the interlayer phase coherence at $\nu=1$. The condensation is a result of intralayer and interlayer Coulomb interactions among electrons in the highly degenerate lowest Landau level. It is easy to see that the condensation naturally leads to Josephson equations [1] governing I-V characteristics of dissipationless currents. We start with Schrodinger equations of electron fields $\Psi_{i}, i \partial_{t} \Psi_{1}=v_{1} \Psi_{1}-\Delta_{\text {sas }} \Psi_{2}$ and $i \partial_{t} \Psi_{2}=v_{2} \Psi_{2}-\Delta_{\text {sas }} \Psi_{1}$, where $v_{i}$ is a 
chemical potential of electrons in the $i$-th layer and $\Delta_{\text {sas }}$ is a tunneling strength. Note that the kinetic term of electrons is quenched by strong magnetic field $B$ perpendicular to the layers. It follows that the Josephson tunneling current is given by

$$
e \partial_{t}<\Psi_{1}^{\dagger} \Psi_{1}>=i e \Delta_{\text {sas }}\left(<\Psi_{1}^{\dagger} \Psi_{2}>-<\Psi_{1} \Psi_{2}^{\dagger}>\right)=2 e \Delta_{\text {sas }} \sqrt{\rho_{1} \rho_{2}} \sin \theta
$$

where we have used the normalization of the field determined from more detail calculations [4.9]; $<\Psi_{1}^{\dagger} \Psi_{2}>\simeq \sqrt{\rho_{1} \rho_{2}} e^{-i \theta}$. Similarly, we obtain the other equation governing the development of the phase $\theta, \partial_{t} \theta=\left(v_{1}-v_{2}\right)=e V$. Therefore, it is very natural to expect that Josephson effects arise in this coherent bilayer quantum Hall system. In the above derivation, however, the effect of pseudoSkyrmions is not included. As far as the filling factor is exactly equal to 1, such Skyrmion excitations are absent in the quantum Hall states. But, in general, the Skyrmions are present in the state with $\nu \neq 1$. It seems apparently that their effects are negligible because their number is relatively so small that the effects are of the order of $\delta \nu=\nu-1$; we can choose any small values of $\delta \nu$, e.g. $1 / 100$. We should mention, however, that the phase coherence is needed for dissipationless tunneling current, but the presence of the pseudoSkyrmions disturbs the coherence because of their distorted phase configuration. Therefore, even if the number of the pseudoSkyrmions are negligibly small, we need to take account of their effects on the conductivity.

As we have shown in the previous paper [10], solutions of the pseudoSkyrmions in bosonized electron theory [6] of quantum Hall states are given by

$$
\Psi_{1}=\sqrt{\rho_{1}}\left(z+c_{1}\right) \exp (-a(r)) \exp \left(i \theta_{1}\right) \quad \text { and } \quad \Psi_{2}=\sqrt{\rho_{2}}\left(z+c_{2}\right) \exp (-a(r)) \exp \left(i \theta_{2}\right)
$$

with $z=x-i y$ and $r=|z|$, where bosonized electron field $\Psi_{i}$ in the i-th layer goes to, $\sqrt{\rho_{i}} \exp \left(\theta_{i}\right)$, as $r \rightarrow \infty ; \theta=\theta_{1}-\theta_{2}$. The function $a(r)$ is approximately given by $\exp (-a(r)) \simeq \sqrt{1 /\left(r^{2}+c^{2}\right)}$ for $c>l$. Here the parameter $c$ represents length scale of the pseudoSkyrmion; $c_{1}=c \sqrt{\rho_{2} / \rho_{1}}$ and $c_{2}=-c \sqrt{\rho_{1} / \rho_{2}}$. The scale is determined by minimizing the energy of the pseudoSkyrmions,

$$
E_{p s k}=4 \pi \rho_{s s}+\frac{0.46 e^{2}}{\epsilon c}+\frac{0.4 e^{2} \sigma^{2}}{\epsilon l} \frac{d}{l} \frac{c^{2}}{l^{2}}+\frac{\Delta_{s a s} \sqrt{1-\sigma^{2}}}{2 \pi} \frac{c^{2}}{l^{2}}
$$


with $\rho_{s s} \simeq 0.005\left(1-\sigma^{2}\right) e^{2} / \epsilon l$ for $d / l=1.5$ and dielectric constant $\epsilon$. The first term represents exchange energy of pseudo-spin, the second one does Coulomb energy, the third one does charging energy and the final term represents tunneling energy. Thus, the typical scale of pseudoSkyrmion $c$ is given by

$$
c \simeq l\left\{\frac{0.46 e^{2} / 2 \epsilon l}{\Delta_{\text {sas }} \sqrt{1-\sigma^{2}}+\left(0.4 e^{2} \sigma^{2} / \epsilon l\right)(d / l)}\right\}^{1 / 3}
$$

Later, we will determine the scale $c$ by using the data [3] in the observation of the $B_{\|}$ dependence of the tunneling conductivity. Then, we will find that $c \sim 10 l$ and $\sigma \sim 0.01$.

The pseudoSkyrmion has positive electric charge $|e|$ and is present in the region of $\delta \nu<0$, on the other hand, anti-pseudoSkyrmion has negative charge - $|e|$ and is present in the region of $\delta \nu>0$. Speculated from the fact that it possesses the charging energy, the electric charge of the pseudoSkyrmion on 1 st layer is different from that on 2 nd layer when $\rho_{1}-\rho_{2} \neq 0$. Hence, it produces a polarization field, $E_{p}$, perpendicular to the layers,

$$
E_{p}(\vec{x})=\frac{1}{d \epsilon} \int d y^{2}\left(\frac{1}{|\vec{x}-\vec{y}|}-\frac{1}{\sqrt{(\vec{x}-\vec{y})^{2}+d^{2}}}\right)\left(\rho_{1}(\vec{y})-\rho_{2}(\vec{y})\right)
$$

where $\rho_{1}(\vec{y})=e\left(\left|\Psi_{1}\right|^{2}-\rho_{1}\right)$ and $\rho_{2}(\vec{y})=e\left(\left|\Psi_{2}\right|^{2}-\rho_{2}\right)$ with $\Psi_{i}$ given in eq(2) .

This electric field dissipates the energy of the tunneling current and gives rise to a finite conductivity. To see it we note that the interlayer tunneling current $J$ involving the effect of a pseudoSkyrmion is given by

$$
\begin{aligned}
J(\vec{x}) & =e \Delta_{\text {sas }} \rho \sqrt{1-\sigma^{2}} \exp (-2 a(r))\left\{\left(r^{2}-c^{2}-2 c x \frac{\sigma}{\sqrt{1-\sigma^{2}}}\right) \sin \theta+\right. \\
& \left.+2 c y\left(\frac{1}{\sqrt{1-\sigma^{2}}}\right) \cos \theta\right\},
\end{aligned}
$$

where we have used the formula in eq(11) and the solutions in eq(21). Then, it follows that the rate $w$ of the energy dissipation is

$$
w=\int d x^{2} d E_{p}(\vec{x}) J(\vec{x})=2 e^{2} \pi^{2} \sigma \Delta_{s a s} c^{2} \rho^{3 / 2} f\left(c^{2} / l^{2}, d^{2} / l^{2}\right) \sin \theta / \epsilon
$$

where we have taken only the term with the order of $\sigma$ in the limit as $\sigma \rightarrow 0$ and $f\left(c^{2} / l^{2}, d^{2} / l^{2}\right)$ is numerically of the order of 0.1 for $c \simeq 10 l, d \simeq 2 \times 10^{-6} \mathrm{~cm}$ and 
$\rho \simeq 5 \times 10^{10} / \mathrm{cm}^{2}$. We find that the dissipation only arises at $\sigma \neq 0$. It results from the fact that the pseudoSkyrmion possesses polarization only when $\sigma \neq 0$. Actually, the charging energy in eq(3) vanishes at $\sigma=0$.

We now calculate interlayer tunneling conductivity in the presence of $N_{0}$ pseudoSkyrmions. We suppose that the contribution of each pseudoSkyrmion is incoherently taken into account. Then, The total rate $W$ of the energy dissipation is $N_{0} w$, which is equal to the product, $W=V I$, of the everage voltage $V$ and the total tunneling current $I=I_{0}+I_{s}=\int d x^{2}\left(J_{0}+N_{0} J_{s}(\vec{x})\right)$, where the first term $I_{0}$ represents the contribution of the groundstate and the second term $I_{s}$ represents the contribution of the pseudoSkyrmions; $J_{0}=2 e \sqrt{\rho_{1} \rho_{2}} \Delta_{\text {sas }} \sin \theta, J_{s}(\vec{x})=J(\vec{x})-J_{0}$ and $I_{s}=-2 \pi N_{0} e \Delta_{\text {sas }} \rho c^{2} \sqrt{1-\sigma^{2}} k_{0} \sin \theta\left(k_{0}\right.$ is a numerical constant ). Then, the conductivity $G=\partial I / \partial V$ is approximately given by

$$
G \simeq \frac{I}{V}=\frac{\left(I_{0}+I_{s}\right)^{2}}{N_{0} w}=\frac{\Delta_{\text {sas }} N\left(1-2 \pi \rho c^{2} k_{0} \delta \nu\right)^{2} \epsilon \sin \theta}{2 \pi^{2} c^{2} \sigma \rho^{3 / 2} f \delta \nu} \sim 6 \times 10^{-5} \Omega^{-1} \frac{\left(1-2 \pi \rho c^{2} k_{0} \delta \nu\right)^{2} \sin \theta}{f \sigma \delta \nu}
$$

in the limit of $\sigma \rightarrow 0$, where we have used the parameters taken in the experiment [1], $d / l \simeq 1.6, \Delta_{\text {sas }} \simeq 0.1 \mathrm{mK}$ and surface area $\left(\simeq(0.2 \mathrm{~mm})^{2}\right)$ occupied by two dimensional electrons. $N=\int d x^{2} \rho$ denotes total number of electrons; $\delta \nu=N_{0} / N$ and $k_{0}$ is of the order of 1 .

Although the absolute value of $G$ is much larger than that of the observation, the dependence on $\delta \nu$ agrees roughly with the observation. Even if we take $\delta \nu$ to be 0 , there would be residual pseudoSkyrmions. It seems that such residual excitations induce finite conductivity observed around $\nu=1$ [1], although there is a possibility that other mechanisms of the current dissipation still work. We note that the dependence of $G$ on the imbalance parameter $\sigma$ is the same as that on $\delta \nu$. It may be easy to check the dependence observationally. We also note that in our formula both of $G$ and $I$ are proportional to $\sin \theta$. Therefore, it is interesting to check this point by an experiment with current feed.

Now, let us consider the dependence of the conductivity on parallel magnetic field $B_{\|}$ pointing to $y$ direction. The effect is taken only by changing the phase factor $\theta$ in the 
currents $J$ in eq(6) and $J_{0}$ such that $\theta \rightarrow \theta+Q x$ with $Q=e d B_{\|}$; the polarization field $E_{p}$ is not changed. Then, we see that the current $I_{s}(Q)=\int d x^{2} J_{s}(\vec{x}, Q)$ involving the effect of pseudoSkyrmion decays such as $I_{s}(Q) \rightarrow \exp (-Q c)$ for $Q \rightarrow \infty$. We also note that the groundstate current $I_{0}(Q)=\int d x^{2} J_{0}(Q)$ behaves such as $I_{0}(Q) \propto \sin \left(e \Phi / \Phi_{0}\right) /\left(e \Phi / \Phi_{0}\right)$ where $\Phi=d \int d x B_{\|}$denotes flux penetrating the region between the two layers; $\Phi_{0}=$ $e / \pi$. This term is negligibly small even for $B_{\|}=0.1 \mathrm{~T}$, while the other term $I_{s}(Q)$ is not small for such $B_{\|}$. Therefore, the conductivity behaves such that $G(Q) \sim\left(I_{0}(Q)+\right.$ $\left.I_{s}(Q)\right)^{2} /\left(N_{0} w(Q)\right) \sim I_{s}(Q)^{2} /\left(N_{0} w(Q)\right) \rightarrow \exp (-Q c)$ as $Q$ becomes large. Comparing this one with the observation, we can determine the scale of pseudoSkyrmion, $c \sim 10 l$. This value agrees roughly with the one derived with a different method [12]. We find that the pseudoSkyrmion in the observation is large so that even if we take $\delta \nu=1 / 100$, the whole plain is occupied by the pseudoSkyrmions.

We stress that not only the presence of the zero bias peak even at small $B_{\|} \neq 0$, but also the rapid decay of the peak as $B_{\|}$going beyond $\sim 0.1$ Tesla, can be explained as the effect of the pseudoSkyrmions. This fact strongly suggests that the finite interlayer tunneling conductivity in the recent observation is caused mainly by the pseudoSkyrmions, although our estimation of the conductivity is much larger than that of the observation.

Finally we wish to point out that in order to check our conclusion and observe dissipationless Josephson tunneling current, the imbalance $\sigma=\left(\rho_{1}-\rho_{2}\right) / \rho$ should be diminished with carefully adjusting bias voltage between the two layers. Then, we expect that the balance state $\sigma=0$ would be realized automatically when $\sigma$ is adjusted to be less than a critical one, and we will be able to see Josephson effects in the quantum Hall states even in the presence of residual pseudoSkyrmions.

We thank F.Z. Ezawa and A. Sawada for fruitful discussions and members of theory group in KEK for their hospitality. 


\section{REFERENCES}

[1] I.B. Spielman, J.P. Eisenstein, L.N. Pfeiffer and K.W. West, Phys. Rev. Lett. 84, 5808 (2000).

[2] L. Balents and L. Radzihovsky, Phys. Rev. Lett. 86, 1825 (2001); A.S. Stern, S.M. Girvin, A.H. MacDonald and N.M. Ma, Phys. Rev. Lett, 86, 1829 (2001); M.M. Fogler and F. Wilczek, Phys. Rev. Lett, 86, 1833 (2001).

[3] I.B. Spielman, J.P. Eisenstein, L.N. Pfeiffer and K.W. West, Phys. Rev. Lett. 87, 036803 (2000); cond-mat/0108043

[4] Z.F. Ezawa and A. Iwazaki, Int. J. Mod. Phys. B 19, 3205 (1992); Phys. Rev. B 47, 7295 (1993), A. Iwazaki, Mod. Phys. Lett. B12, 327 (1998), X.G. Wen and A. Zee, Phys. Rev. Lett. 69, 1811 (1992).

[5] S.C. Zhang, T.H. Hansen and S. Kivelson, Phys. Rev. Lett. 62, 82 (1989)

[6] Z.F. Ezawa, M. Hotta and A. Iwazaki, Phys. Rev. B46, 7765 (1992); Z.F. Ezawa and A. Iwazaki, Phys. Rev. B43, 2637 (1991).

[7] Z.F. Ezawa and A. Iwazaki, Phys. Rev. B47, 7295 (1993); Int. J. Mod. Phys. B8, 2111 (1994), references therein.

[8] S.Q. Murphy, J.P. Eisenstein, G.S. Boebinger, L.N. Pfeiffer and K.W. West, Phys. Rev. Lett. 72, 728 (1994), A. Sawada, Z.F. Ezawa, H. Ohno, Y. Horikoshi, Y. Ohno, S. Kishimoto, F. Matsukura, M. Yasumoto and A. Urayama, Phys. Rev. Lett. 80, 4534 (1998), A. Sawada, Z.F. Ezawa, H. Ohno, Y. Horikoshi, A. Urayama, Y. Ohno, S. Kishimoto, F. Matsukura, M. Yasumoto and N. Kumada, Phys. Rev. B 59, 14888 (1999).

[9] K, Yang et al, Phys. Rev. Lett. 72, 732 (1994), K, Moon et al, Phys. Rev. B 51, 5138 (1995), M. Abolfath, L. Radzihovsky and A.H. MacDonald, cond-mat/0110049, Y.N. Joglehar and A.H. MacDonald, cond-mat/0111056. 
[10] A. Iwazaki, Phys. Rev. B 65, 153307 (2002).

[11] S.L. Sondhi, A. Karlhede, S.A. Kivelson and E.H. Rezayi, Phys. Rev. B 47, 16419 (1993).

[12] K. Moon and K. Mullen, Phys. Rev. B 57, 1378 (1998). 